\title{
Undecidable theories of Lyndon algebras
}

\author{
Vera Stebletsova* $\quad$ Yde Venema ${ }^{\dagger}$
}

\begin{abstract}
With each projective geometry we can associate a Lyndon algebra. Such an algebra always satisfies Tarski's axioms for relation algebras and Lyndon algebras thus form an interesting connection between the fields of projective geometry and algebraic logic. In this paper we prove that if $\mathrm{G}$ is a class of projective geometries which contains an infinite projective geometry of dimension at least three, then the class $L(G)$ of Lyndon algebras associated with projective geometries in $G$ has an undecidable equational theory. In our proof we develop and use a connection between projective geometries and diagonal-free cylindric algebras.
\end{abstract}

\section{Introduction}

Lyndon algebras form an interesting connection between the fields of projective geometry and algebraic logic. Their origin lies in the paper [Jónsson 1959] where the author associates a relation algebra with a projective plane. Jónsson's construction was generalized by R. Lyndon to projective geometries of arbitrary dimension, in order to provide "a method for deriving consequences in the algebraic theory of binary relations from certain familiar facts of projective geometry", cf. [Lyndon 1961].

We need to go into some detail concerning this construction. We formalize a projective geometry as a two-sorted structure $\mathcal{G}=(P, L, I)$ where $P$ is a set of points, $L$ is a set of lines, and $I \subseteq P \times L$ is the binary incidence relation (precise definitions will be given in the next section). Now the Lyndon algebra associated with a projective geometry $\mathcal{G}=(P, L, I)$ is the algebraic structure $\mathcal{L}(\mathcal{G})=\left(\wp\left(P_{e}\right), \cup, \sim, \varnothing, \circ, \iota,\{e\}\right)$ defined as follows. The carrier of $\mathcal{L}(\mathcal{G})$ is the power set of the set $P_{e}=P \cup\{e\}$ - here $e$ is some new, 'useless' point; the operations $\cup$ and $\sim$ denote the ordinary set-theoretic operations of union and complement (with respect to $\left.P_{e}\right)$, respectively, and $\varnothing$ denotes the empty set. The interesting operation of Lyndon algebras is $\circ$ which interprets the binary operator symbol ; (in algebras of relations corresponding to relational composition). We first define this operation $\circ$ for the atoms of the algebras; that

${ }^{*}$ Department of Philosophy, Utrecht University, Heidelberglaan 8, 3584 CS Utrecht, the Netherlands. Email: Vera.Stebletsova@phil.uu.nl. The research of this author is sponsored by the Netherlands Organization for the Advancement of Research (NWO).

${ }^{\dagger}$ Institute for Logic, Language and Computation, University of Amsterdam, Plantage Muidergracht 24, NL-1018 TV Amsterdam, The Netherlands. E-mail: yde@wins.uva.nl. The research of this author has been made possible by a fellowship of the Royal Netherlands Academy of Arts and Sciences. 
is, singleton subsets of $P_{e}$ (here $\overline{p q}$ denotes the line through $p$ and $q$ ). This definition extends to arbitrary subsets of $P_{e}$ by requiring complete additivity for $\circ$.

$$
\begin{aligned}
\{p\} \circ\{q\} & = \begin{cases}\{e, p\} & \text { if } p=q, \\
\overline{p q} \backslash\{p, q\} & \text { if } p \neq q \text { and } e \notin\{p, q\}, \\
\{p\}, & \text { if } q=e, \\
\{q\}, & \text { if } p=e .\end{cases} \\
X \circ Y & =\bigcup_{p \in X, q \in Y}\{p\} \circ\{q\}
\end{aligned}
$$

The operation $\iota$ is there only to give Lyndon algebras the right similarity type, namely that of Tarski's relation algebras; it interprets the unary function symbol ${ }^{\smile}$ (corresponding to taking the converse of a relation) and is trivially defined as the identity map: $\iota(X)=X$ for any subset $X$ of $P_{e}$. Finally, the singleton $\{e\}$ interprets the constant 1' (which is the symbol denoting the identity relation in algebras of real binary relations).

Given a class $\mathrm{G}$ of projective geometries, we denote by $\mathrm{L}(\mathrm{G})$ the class of associated Lyndon algebras. In a number of occasions we will be interested in the Lyndon algebras associated with the class $\mathrm{G}_{d}$ of all $d$-dimensional projective geometries ( $d$ some fixed cardinal); the class of these $d$-dimensional Lyndon algebras is denoted as $\mathrm{L}_{d}$.

Lyndon algebras have played an important role in the representation theory of relation algebras. First of all, it is straightforward to show that every Lyndon algebra is indeed a relation algebra; that is, it satisfies the axioms that Tarski proposed as a first approximation for an axiomatization of the 'true' relational equations. In [Jónsson 1959] it is proved that if a plane does not satisfy Desargue's Theorem, then its associated Lyndon algebra is not representable; this result provided natural examples of non-representable relation algebras. Although Lyndon generalized Jónsson's work to geometries of arbitrary dimension, his paper [Lyndon 1961] is usually cited for his work on algebras associated with lines (one-dimensional projective geometries); in fact, some people use the term 'Lyndon algebras' only for this smaller class of algebras. An important result of Lyndon's paper states that for a line $\mathcal{G}$ of $n$ points $(n \geq 4)$, the algebra $\mathcal{L}(\mathcal{G})$ is representable iff there is a projective plane in which all lines have $n$ points. This result formed the basis of Monk's seminal theorem that the equational theory of the class RRA is not finitely axiomatizable, cf. [Monk 1964].

Some further results are known about Lyndon algebras. For instance, Andréka, Givant and Németi proved interesting results on the decidability of various classes of one-dimensional Lyndon algebras, cf. [Andréka, Givant \& Németi 1997]. In particular, it is proved that the class $L_{1}$ itself has a decidable equational theory. (The cited paper forms an excellent overview of the state of the art concerning decidability and undecidability of various classes of relation algebras.) For some natural classes of geometries, [Givant 199?] contains results connecting the associated Lyndon algebras to the universal class of simple algebras in the variety generated by $L(G)$. Finally, the paper [Stebletsova 1998] is concerned with the axiomatizability problem of various classes of Lyndon algebras; the main result of that paper is a finite axiomatization for the equational theory of $\mathrm{L}_{d}$ for fixed, finite $d$.

In this paper we investigate the decidability of the equational theories of Lyndon algebras of higher dimension. Our main result is the following theorem. 
Theorem 1 If $\mathrm{G}$ is a class of projective geometries which contains an infinite projective geometry of dimension at least three, then the equational theory of $\mathrm{L}(\mathrm{G})$ is undecidable.

In particular, the class of all Lyndon algebras has an undecidable equational theory, and so have the classes $\mathrm{L}_{d}$ for $d \geq 3$.

Two remarks are in order concerning our proof of Theorem 1. First, it turns out that in order to reach undecidability, we do not need the full expressive power of the extra-boolean operators of Lyndon algebras. We define the slender Lyndon algebra $\mathcal{S}(\mathcal{G})$ of a projective geometry $\mathcal{G}=(P, L, I)$ to be the structure $\mathcal{S}(\mathcal{G})=(\wp(P), \cup, \sim, \varnothing, \bullet)$. Here $(\wp(P), \cup, \sim, \varnothing)$ is the power set algebra over $P$, on which the binary operation $\bullet$ on $\wp(P)$ is defined as follows (again, first for singletons and then for arbitrary subsets of $P$ ).

$$
\begin{aligned}
\{p\} \bullet\{q\} & = \begin{cases}\overline{p q} & \text { if } p \neq q \\
\{p\} & \text { if } p=q,\end{cases} \\
X \bullet Y & =\bigcup_{p \in X, q \in Y}\{p\} \bullet\{q\}
\end{aligned}
$$

In the next section we will see that when $X$ and $Y$ are subspaces of the geometry, then $X \bullet Y$ is in fact the subspace generated by $X$ and $Y$. For a class $\mathrm{G}$ of projective geometries, we let $\mathrm{S}(\mathrm{G})$ denote the class of slender Lyndon algebras of geometries in $\mathrm{G}$; we will use the notation $S$ and $S_{d}$ with obvious meanings. Our main technical result (Theorem 3.1 below) states that for any class $G$ containing an infinite projective geometry of dimension at least three, the equational theory of the class $\mathrm{S}(\mathrm{G})$ is undecidable. As we will show in section 3 , it is straightforward to derive Theorem 1 from this; the crucial observation in this proof is that the operation $\bullet$ can be defined in terms of $\circ$ and the other operations of Lyndon algebras.

Second, the main idea underlying our proof involves an interesting connection with another kind of algebras that are well-known from algebraic logic, namely the diagonal-free cylindric algebras (cf. the next section for definitions). Roughly speaking, we will show that inside every slender Lyndon algebra associated with a projective geometry of dimension at least three, we can find a term-definable representable diagonal-free cylindric algebra of dimension three (a representable $\mathrm{Df}_{3}$, for short). And conversely, given a 'small', simple representable $\mathrm{Df}_{3} \mathcal{A}$ and an infinite geometry $\mathcal{G}$ of dimension at least three, we can embed $\mathcal{A}$ in one of these representable $\mathrm{Df}_{3}$ 's living inside the slender Lyndon algebra associated with $\mathcal{G}$. These connections, which modify and generalize an idea of [Venema 1998], allow us to use R. Maddux' result on the undecidability of the equational theory of the class of representable $\mathrm{Df}_{3}$ 's [Maddux 1980] in order to prove our main technical result.

Finally, we want to mention that our results leave open an interesting problem, namely whether the class $L_{2}$ of Lyndon algebras associated with projective planes has a decidable equational theory. S.Givant (personal communication) announced some strengthenings of our main result; these will be briefly mentioned at the end of the paper.

Acknowledgement We would like to thank the anonymous referee for a very careful reading of the manuscript of this paper, and for making many suggestions for improving its presentation. 


\section{Preliminaries}

We will assume a certain familiarity with the basics of geometry and universal algebra. We now fix our notation and terminology, and we gather some specific facts from the fields of projective geometry and diagonal-free cylindric algebras that we need in our proofs. Since Lyndon algebras are the only relation algebras that we will encounter here, we do not supply further information on this branch of algebraic logic. The interested reader is referred to [Jónsson 1982] or [Németi 1991].

\section{Universal algebra}

Given a term $\tau=\tau\left(x_{1}, \ldots, x_{n}\right)$ in an algebraic similarity type and an algebra $\mathcal{A}$ of this similarity type, $\tau$ induces a map: $A^{n} \rightarrow A$. This map is denoted by $\tau^{\mathcal{A}}$.

In this paper we will be dealing with three different similarity types, namely those of relation algebras/Lyndon algebras, slender Lyndon algebras and three-dimensional diagonalfree cylindric algebras. For brevity, we will denote the second similarity type by $S L$ and the third by $D f_{3}$. All these similarity types extend that of Boolean algebras; for the boolean reducts of the similarity types we will use uniform and familiar notation like $+,-, 0, \cdot$ and 1. Of these, we consider the first three as primitive and the other two as abbreviations. The relation algebraic similarity type has a binary function symbol ; a unary $\smile$ and a constant 1'; as abbreviation we use 0 ' for -1 '. The similarity type $S L$ has only one non-boolean function symbol; it is binary and we also denote it by ;. Three-dimensional diagonal-free cylindric algebras will be discussed below.

For all these languages, we will use standard abbreviations for certain formulas, such as $\sigma \preceq \tau$ for $\sigma+\tau \approx \tau, \sigma \not \nsim \tau$ for $\neg(\sigma \approx \tau)$, and $\sigma \prec \tau$ for $\sigma \preceq \tau \wedge \sigma \not \approx \tau$.

\section{Projective geometries}

As we mentioned before, we model projective geometries as structures $\mathcal{G}=(P, L, I)$, where $P$ is a non-empty set of points, $L$ is a non-empty set of lines, and $I \subseteq P \times L$ is a binary incidence relation between points and lines. We use standard geometrical terminology like 'two lines meet in the point $s$ '; we may also follow standard practice in identifying a line with the set of points that are incident with it. Now such a two-sorted structure $\mathcal{G}$ is a projective geometry or projective space if it satisfies the following axioms:

(A1) There exists at least one line, and each line has at least four points;

(A2) Each pair of distinct points $(x, y)$ lies on a unique line, $\overline{x y}$;

(A3) If $x, y$ and $z$ are distinct points, and a line meets lines $\overline{x y}$ and $\overline{x z}$ in distinct points, then it meets $\overline{y z}$.

In the sequel, we will frequently omit the adjective 'projective' when referring to geometries.

Now let $\mathcal{G}=(P, L, I)$ be a geometry. A subset $S$ of $P$ is a subspace of $\mathcal{G}$ if for all distinct points $p, q$ in $S$, we have $\overline{p q} \subseteq S$. For any $X \subseteq \mathcal{G}$, define $\langle X\rangle$, the span of $X$ or subspace generated by $X$, to be the smallest subspace containing $X$. It is easy to see that $\langle X\rangle$ is the intersection of all subspaces containing $X$. A hyperplane $H$ of a geometry $\mathcal{G}$ is a maximal 
proper subspace of $\mathcal{G}$. If $\langle X\rangle=S$ we say that $X$ is a spanning set of $S$. We write $\left\langle X_{1}, \ldots, X_{n}\right\rangle$ for $\left\langle X_{1} \cup \ldots \cup X_{n}\right\rangle$, and $\langle p, q, r\rangle$ for $\langle\{p\},\{q\},\{r\}\rangle$. A subset $S$ of $\mathcal{G}$ is independent if for all $p \in S$ we have $\langle S \backslash\{p\}\rangle \neq\langle S\rangle$. An independent spanning set of $\mathcal{G}$ is called a basis of $\mathcal{G}$. Every geometry has a basis and all bases of a geometry have the same cardinality; this cardinal is called the rank $r$ of $\mathcal{G}$. Finally, we can define the dimension $d$ of $\mathcal{G}$ as $d=r-1$ if $r$ is finite, and $d=\infty$ if $r$ is infinite. One-dimensional projective spaces are called lines, and two-dimensional ones, planes.

One can prove that all lines of a projective space have the same cardinality; a geometry is said to have order $\alpha$ if each of its lines contains $\alpha+1$ points.

For explicit reference we list the following geometric facts. These results are very easy to derive from the definitions, or can be found in any textbook on projective geometry; for instance, the reader might consult Chapter 2 and 6 of [Garner 1981].

Fact 2.1 Let $\mathcal{G}=(P, L, I)$ be a projective geometry. Then

(1) If $S$ is a subspace of dimension 2 of $\mathcal{G}$, i.e., a plane, then any two of its lines meet.

(2) Every line in $\mathcal{G}$ meets every hyperplane $H$ of $\mathcal{G}$.

(3) Let $S_{0}, S_{1}$ be non-empty subspaces of $\mathcal{G}$. Then

$$
\left\langle S_{0}, S_{1}\right\rangle=\left(S_{0} \cap S_{1}\right) \cup \bigcup\left\{\overline{s_{0} s_{1}} \mid s_{0} \in S_{0}, s_{1} \in S_{1} \text { and } s_{0} \neq s_{1}\right\} .
$$

(4) If $S_{i}, S_{j}$ are subspaces of $\mathcal{G}$, then $S_{i} \bullet S_{j}=\left\langle S_{i}, S_{j}\right\rangle$.

(5) For subspaces $S_{0}, S_{1}$ and $S_{2}$ of $\mathcal{G}$ we have $\left\langle S_{0}, S_{1}, S_{2}\right\rangle=\left\langle S_{0},\left\langle S_{1}, S_{2}\right\rangle\right\rangle$.

(6) If a line intersects a subspace, while it does not form part of the subspace, then the intersection is a singleton point.

(7) Let $M$ be an independent set, and $\left\{M_{i}: i<3\right\}$ a partition of $M$. Then there are no collinear points $s_{0} \in\left\langle M_{0}\right\rangle, s_{1} \in\left\langle M_{1}\right\rangle$ and $s_{2} \in\left\langle M_{2}\right\rangle$.

\section{Boolean algebras with operators}

All algebras that we encounter in this paper are Boolean algebras with operators; that is, Boolean algebras augmented with operations that are additive in each of their arguments. The prime examples of such algebras are the so-called complex algebras.

Given a set $W$ and a relation $R \subseteq W^{n+1}$, define the operation $m_{R}: \wp(W)^{n} \rightarrow \wp(W)$ by $m_{R}\left(X_{1}, \ldots, X_{n}\right)=\left\{y \in W \mid R y x_{1} \ldots x_{n}\right.$ for some $\left.x_{1} \in X_{1}, \ldots, x_{n} \in X_{n}\right\}$. The complex algebra of a relational structure $\mathcal{F}=\left(W, R_{i}\right)_{i \in I}$ is defined as the algebra $\mathcal{F}^{+}=\left(\wp(W), \cup, \sim, \varnothing, m_{R_{i}}\right)_{i \in I}$, with $(\wp(W), \cup, \sim, \varnothing)$ being the power set algebra of $W$. The structure $\mathcal{F}$ is called the atom structure of $\mathcal{F}^{+}$; we will frequently deal with properties of complex algebras that correspond to certain properties of their atom structures.

A class $\mathrm{K}$ of Boolean algebras with operators is a discriminator class if there is some unary term $\sigma(x)$ such that $\mathrm{K}=x \not \approx 0 \rightarrow \sigma(x) \approx 1$ and $\mathrm{K} \models \sigma(0)=0$.

Fact 2.2 (Cf. [Tarski \& Givant 1988]) For any discriminator class K of Boolean algebras with operators there is a recursive translation which turns any universal formula into an equation which is equivalent to it over $\mathrm{K}$. 


\section{Diagonal-free cylindric algebras}

Diagonal-free cylindric algebras, and in particular, the representable ones, form an algebraic counterpart of first-order logic without equality. For a survey of the theory of these algebras the reader is referred to Chapter 5 of [Henkin, Monk \& Tarski 1985]; here we confine ourselves to the definitions and those facts that we need later on. Let $\alpha$ be an arbitrary but fixed ordinal.

A diagonal-free cylindric algebra of dimension $\alpha$, for brevity a $\operatorname{Df}_{\alpha}$, is an algebra $\mathcal{A}=$ $\left(A,+, \cdot,-, 0, \mathrm{c}_{i}\right)_{i \in \alpha}$ such that $(A,+, \cdot,-, 0)$ is a boolean algebra, and each $\mathrm{c}_{i}$ is a unary operation on $A$ such that the following identities hold in $\mathcal{A}$, for all $i, j \in \alpha$ :

(C1) $\mathrm{c}_{i} 0 \approx 0$

(C2) $x \preceq \mathrm{c}_{i} x$, (i.e., $x+\mathrm{c}_{i} x \approx \mathrm{c}_{i} x$ )

(C3) $\mathrm{c}_{i}\left(x \cdot \mathrm{c}_{i} y\right) \approx \mathrm{c}_{i} x \cdot \mathrm{c}_{i} y$

(C4) $\mathrm{c}_{i} \mathrm{c}_{j} x \approx \mathrm{c}_{j} \mathrm{c}_{i} x$.

The variety of all diagonal-free cylindric algebras of dimension $\alpha$ is denoted by $\operatorname{Df}_{\alpha}$.

In this article we are mainly interested in the representable $\mathrm{Df}_{\alpha}$ 's; elements of these algebras represent $\alpha$-ary relations. For an arbitrary family $U=\left\{U_{i}: i<\alpha\right\}$ of non-empty sets, we define, for any $i<\alpha$, the relation $\equiv_{i}$ on the Cartesian product $\prod_{i<\alpha} U_{i}$ by

$$
u \equiv_{i} v \text { iff } u_{j}=v_{j} \text { for all } j \neq i
$$

that is, the $\alpha$-sequences $u$ and $v$ are $i$-related if and only if they differ at most in their $i$-th coordinate. Relational structures of the form $\left(\prod_{i<\alpha} U_{i}, \equiv_{i}\right)_{i \in I}$ are called blocks; if $U_{i}=U_{j}$ for every pair $i, j \in I$ we call the structure a cube. Any subalgebra of the structure

$$
\mathcal{C}_{\alpha}(U)=\left(\prod_{i<\alpha} U_{i}, \equiv_{i}\right)_{i \in I}^{+}
$$

is called an $\alpha$-dimensional diagonal-free cylindric set algebra with base system $U$, or shortly: a set $\mathrm{Df}_{\alpha}$ over $U$. Set $\mathrm{Df}_{\alpha}$ 's of the kind $\mathcal{C}_{\alpha}(U)$ are called full. A full set $\mathrm{Df}_{\alpha}$ is countably based if it is of the form $\mathcal{C}_{\alpha}(U)$ with each $U_{i}$ being a countable set. Finally, a $\mathrm{Df}_{\alpha}$ is called representable if it is isomorphic to a subalgebra of a product of full set $\mathrm{Df}_{\alpha}$ 's. The class of such algebras is denoted by $\operatorname{RDf}_{\alpha}$.

From now we concentrate on the case $\alpha=3$. An element of a $\operatorname{Df}_{3} \mathcal{A}$ is called rectangular if it satisfies $a=\mathrm{c}_{0} \mathrm{c}_{1} a \cdot \mathrm{c}_{0} \mathrm{c}_{2} a \cdot \mathrm{c}_{1} \mathrm{c}_{2} a ; \mathcal{A}$ is called rectangularly atomic if it is atomic and each of its atoms is rectangular.

Proofs of the following facts can be found in Chapter 5 of [Henkin, Monk \& Tarski 1985].

Fact 2.3 The following hold for diagonal-free cylindric algebras:

(1) $\mathrm{A} \mathrm{Df}_{3}$ is simple if and only if it satisfies $\forall x\left(x \neq 0 \rightarrow \mathrm{c}_{0} \mathrm{c}_{1} \mathrm{c}_{2} x=1\right)$.

(2) A representable $\mathrm{Df}_{3}$ is simple if and only if it is isomorphic to a set $\mathrm{Df}_{3}$.

(3) Any rectangularly atomic $\mathrm{Df}_{3}$ is representable. If such an algebra is in addition complete and simple, it is isomorphic to a full set $\mathrm{Df}_{3}$.

(4) Suppose that $\mathcal{A}$ is a set $\mathrm{Df}_{3}$ with base system $U$, and $V$ is a larger base system in the sense that $\left|U_{i}\right| \leq\left|V_{i}\right|$ for every $i<3$. Then $\mathcal{A}$ can be embedded in $\mathcal{C}_{3}(V)$.

(5) $\mathrm{RDf}_{3}$ is a variety which is generated by the class of countably based set $\mathrm{Df}_{3}$ 's. 


\section{Proofs}

As we mentioned in our introduction, the main technical result of our paper is the following.

Theorem 3.1 If $\mathrm{G}$ is a class of projective geometries which contains an infinite geometry of dimension at least three, then the equational theory of $\mathrm{S}(\mathrm{G})$ is undecidable.

It is the main aim of this section to prove Theorem 3.1, but let us first see how to derive Theorem 1 from it.

Proof of Theorem 1. Recall that $S L$ denotes the similarity type of slender Lyndon algebras. We will define a translation $\hat{\cdot}$ of $S L$-terms to relation algebraic terms such that for any $S L$-equation $\sigma \approx \tau$, and any geometry $\mathcal{G}$ we have that

$$
\mathcal{S}(\mathcal{G}) \models \sigma \approx \tau \text { iff } \mathcal{L}(\mathcal{G}) \models \widehat{\sigma} \approx \widehat{\tau} .
$$

The definition of this translation proceeds by term induction:

$$
\begin{aligned}
\widehat{x} & =x \cdot 0 \\
\widehat{-\tau} & =-\widehat{\tau} \cdot 0 \\
\widehat{\sigma \cdot \tau} & =\widehat{\sigma} \cdot \widehat{\tau} \\
\widehat{\sigma ; \tau} & =((\widehat{\sigma}+\widehat{\tau}+\widehat{\sigma} ; \widehat{\tau}) \cdot \widehat{\sigma} ; 1 \cdot \widehat{\tau} ; 1) \cdot 0,
\end{aligned}
$$

Since $\widehat{\cdot}$ is a recursive function with a recursive domain, Theorem 1 will follow immediately from (3) and Theorem 3.1.

Let $\mathcal{G}$ be some geometry, and $\tau$ an arbitrary $S L$-term. We claim that

$$
\widehat{\tau}^{\mathcal{L}(\mathcal{G})}\left(Q_{1}, \ldots, Q_{n}\right)=\tau^{\mathcal{S}(\mathcal{G})}\left(Q_{1} \cap P, \ldots, Q_{n} \cap P\right)
$$

for arbitrary subsets $Q_{1}, \ldots, Q_{n}$ of $P_{e}$. This suffices to prove (3) because of the following. Consider an $S L$-equation $\sigma \approx \tau$, and an arbitrary geometry $\mathcal{G}$. First assume that $\mathcal{L}(\mathcal{G}) \equiv$ $\widehat{\sigma} \approx \widehat{\tau}$; we will prove that $\mathcal{S}(\mathcal{G}) \models \sigma \approx \tau$. Consider arbitrary subsets $Q_{1}, \ldots, Q_{n}$ of $P$. It follows from $\mathcal{L}(\mathcal{G})=\widehat{\sigma} \approx \widehat{\tau}$ that $\widehat{\sigma}^{\mathcal{L}(\mathcal{G})}\left(Q_{1}, \ldots, Q_{n}\right)=\widehat{\tau}^{\mathcal{L}(\mathcal{G})}\left(Q_{1}, \ldots, Q_{n}\right)$. Hence, since $Q_{i} \subseteq P$ for all $i,(4)$ gives that $\sigma^{\mathcal{S}(\mathcal{G})}\left(Q_{1}, \ldots, Q_{n}\right)=\tau^{\mathcal{S}(\mathcal{G})}\left(Q_{1}, \ldots, Q_{n}\right)$. But since $Q_{1}, \ldots, Q_{n}$ were arbitrary, this gives that $\mathcal{S}(\mathcal{G}) \models \sigma \approx \tau$, which is what we were after. The other direction is proved in a similar way.

We prove (4) by a straightforward term induction. The only non-trivial part of this proof concerns the case of the induction step when $\tau$ is of the form $\tau_{0} ; \tau_{1}$. In order to cover this case it is sufficient to show that for any $X, Y \subseteq P$ we have that

$$
X \bullet Y=\left((X \cup Y \cup X \circ Y) \cap X \circ P_{e} \cap Y \circ P_{e}\right) \cap P .
$$

For a better understanding of the right hand side of this equation it will help to realize that for any $X \subset P_{e}$,

$$
X \circ P_{e}= \begin{cases}\varnothing & \text { if } X=\varnothing \\ P_{e} & \text { otherwise. }\end{cases}
$$


In other words, $x ; 1$ is a unary discriminator term on the class of Lyndon algebras. The observation (6) makes it easy to see how the parts $X \circ P_{e}$ and $Y \circ P_{e}$ in (5) are needed only to handle the case where one of the sets $X$ and $Y$ is empty.

Now suppose that $p$ belongs to $X \bullet Y$. Then neither $X$ nor $Y$ is empty, and $p \in P$ by definition of $\bullet$, so by (6) it suffices to show that $p$ belongs to the set $X \cup Y \cup X \circ Y$. It follows from the definition of $\bullet$ that there are $x \in X$ and $y \in Y$ such that either $x$ and $y$ are distinct and $p \in \overline{x y}$, or otherwise $p=x=y$. In the first case we have either $p=x$ (and thus, $p \in X$ ), or $p=y$ (and thus, $p \in Y$ ), or else $p \in \overline{x y} \backslash\{x, y\}$, whence $p$ belongs to $\{x\} \circ\{y\}$ and thus to $X \circ Y$. In the second case we immediately obtain that $p \in X$. In either case we find that $p \in X \cup Y \cup X \circ Y$.

For the reverse inclusion, suppose that $p$ is an element of the right hand side of (5). It immediately follows that $X \circ P_{e}$ and $Y \circ P_{e}$ are non-empty, so by (6) $X$ and $Y$ themselves are not empty. Since $p \in X \cup Y \cup X \circ Y$, we may distinguish the following four cases: $p \in X \backslash Y$, $p \in Y \backslash X, p \in X \cap Y$ or $p \in X \circ Y$. In the last two cases $p$ is in $X \bullet Y$ because the latter set is defined to include all lines through distinct points in $X$ and $Y$, as well as all points that are in both $X$ and $Y$. The first two cases are completely symmetric - we confine ourselves to treating the first. That is, assume that $p$ belongs to $X$ but not to $Y$. Since $Y$ is not empty it must contain some element $y$ which is thus distinct from $p$. But then from $p \in \overline{p y}=\{p\} \bullet\{y\}$ it follows that $p \in X \bullet Y$. This proves (5) and hence (4). QED

We now turn to the proof of Theorem 3.1, which is based on three auxiliary results. The first two of these, Lemma 3.3 and Lemma 3.4 below, provide the details of the earliermentioned connection between Lyndon algebras and diagonal-free cylindric set algebras. We then put this connection to work by defining a translation from $D f_{3}$-equations to universal formulas in the algebraic $S L$-language, and proving that any equation is valid in the class of set $\mathrm{Df}_{3}$ 's iff its translation is valid in the class $\mathrm{S}(\mathrm{G})$, for any class $\mathrm{G}$ of geometries that meets the conditions of Theorem 3.1. From this, Theorem 3.1 will follow almost immediately.

Let us first explain the basic idea of the connection, by means of an example. Consider a projective plane $\mathcal{P}=(P, L, I)$, single out one line $h$ which we will call the horizon or line at infinity, and take two distinct points on $h$, say, $s_{0}$ and $s_{1}$. Let $A$ be the set of 'finite' points; that is, $A$ consists of all points not on $h$. Now, given any finite point $p$, we can draw the two lines connecting $p$ with $s_{0}$ and with $s_{1}$ respectively. In the picture below, these lines has been drawn, for various finite points. 


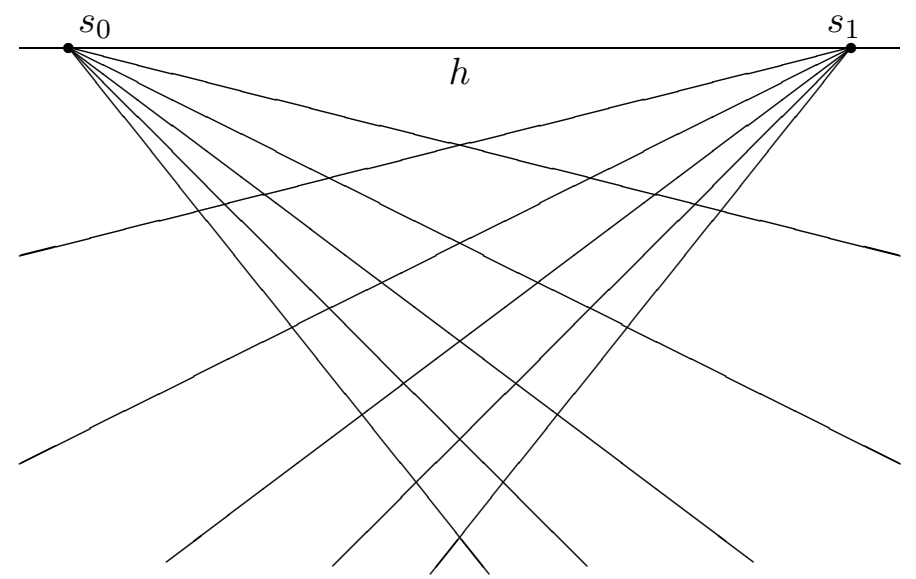

We define the relations $\sim_{0}$ and $\sim_{1}$ on $A$ as follows:

$$
p \sim_{i} q \text { iff } p, q \text { and } s_{i} \text { are collinear. }
$$

That is, $p \sim_{i} q$ if and only if $q$ lies on the line through $p$ 'in the direction of $s_{i}$ '.

The key observation underlying the connection between geometries and diagonal-free algebras is that the relational structure $\left(A, \sim_{0}, \sim_{1}\right)$ is isomorphic to a cube (since we are in dimension 2, the name 'square' would have been more appropriate). In algebraic terms this means that the complex algebra of $\left(A, \sim_{0}, \sim_{1}\right)$ is isomorphic to a full set $\mathrm{Df}_{2}$.

Conversely, consider the complex algebra $\mathcal{A}$ of a block $\left(U_{0} \times U_{1}, \equiv_{0}, \equiv_{1}\right)$, where $U_{0}$ and $U_{1}$ are countable. Now consider an infinite projective plane $\mathcal{P}=(P, L, I)$; that is, assume that $P$ is infinite. This implies that every line has infinitely many points. Hence, carrying out the construction described above (with a line $h \in L$ and points $s_{0}$ and $s_{1}$ on $h$ ), we find that all equivalence classes of $\sim_{0}$ and $\sim_{1}$ are infinite. Hence, the structure $\left(A, \sim_{0}, \sim_{1}\right)$ is isomorphic to a square $\mathcal{B}=\left(U \times U, \equiv_{0}, \equiv_{1}\right)$ based on an infinite set $U$. It follows from (the two-dimensional version of ) Fact 2.3(5) that $\mathcal{A}$ can be embedded in the complex algebra $\mathcal{B}^{+}$, and hence, in the diagonal-free set algebra associated with $\mathcal{P}, h, s_{0}$ and $s_{1}$.

It is obvious how to adapt this idea to projective geometries of dimension three: instead of a line $h$ we take a plane $H$, and instead of two designated 'direction points' in $H$, we take three such points. However, the geometries that we are involved with in this paper are not necessarily of dimension exactly three, but may be of arbitrary dimension (at least three). We modify our construction as follows. Instead of working with direction points $s_{0}, s_{1}$ and $s_{2}$ we work with direction spaces $S_{0}, S_{1}$ and $S_{2}$. To be precise, suppose that $\mathcal{G}$ has a hyperplane $H$ which is spanned by three suitable subspaces $S_{0}, S_{1}$ and $S_{2}$. Let $A$ be the set of 'finite' points, that is, $A=P \backslash H$; and for $i<3$, define the relation $\sim_{i}$ on $A$ by

$$
p \sim_{i} q \text { iff for some } s \in S_{i} \text {, the points } p, q \text { and } s \text { are collinear. }
$$

We can still prove that these relations $\sim_{i}$ are equivalence relations, although their equivalence classes may not all have the same cardinality. Fortunately however, provided that we impose some restrictions on $S_{0}, S_{1}$ and $S_{2}$, we can still make sure that the structure $\left(A, \sim_{i}\right)_{i<3}$ 
is isomorphic to a block. Again phrased in algebraic terms, we can show that with each suitable collection of subspaces $H, S_{0}, S_{1}$ and $S_{2}$, the associated complex algebra $\left(A, \sim_{i}\right)_{i<3}^{+}$ is isomorphic to a full set $\mathrm{Df}_{3}$. And vice versa, given a 'small' set $\mathrm{Df}_{3}$, we can 'build a geometry around its atom structure'.

There is one minor problem left, due to the fact that we want to catch this construction in a nice translation mapping equations in the language of diagonal free algebras to formulas in the algebraic language of slender Lyndon algebras. The point is that although we can express algebraically that an $S L$-variable is interpreted as a subspace of the geometry, we cannot say that it refers to a hyperplane. What happens if we carry out the construction sketched above for an arbitrary subspace $H$, not necessarily a hyperplane? As we will see below, the only difference this makes is that the structure $\left(A, \sim_{i}\right)_{i<3}$ will not be isomorphic to a block, but to a disjoint union of such blocks. Its complex algebra will therefor not be isomorphic to a full set $\mathrm{Df}_{3}$, but to a product of such algebras. This will turn out to be only a minor complication.

Now that we have given an informal explanation of our constructions, let us provide the technical details. The following notion will be used throughout the remainder of the paper.

Definition 3.2 Let $\mathcal{G}$ be a projective geometry with point set $P$. A quadruple $T=\left(H, S_{0}, S_{1}, S_{2}\right)$ is called a tripod in $\mathcal{G}$ if

- $H$ is a non-empty proper subspace of $\mathcal{G}$,

- $S_{0}, S_{1}$ and $S_{2}$ are proper, mutually disjoint subspaces of $H$ that span $H$,

- while there is no triple of collinear points $s_{0} \in S_{0}, s_{1} \in S_{1}$ and $s_{2} \in S_{2}$.

For a tripod $T=\left(H, S_{0}, S_{1}, S_{2}\right)$ in $\mathcal{G}$, we put $A_{T}=P \backslash H$, and define the binary relations $\sim_{i}$ on $A_{T}$ for $i<3$ :

$$
p \sim_{i} q \text { iff for some } s \in S_{i} \text {, the points } p, q \text { and } s \text { are collinear. }
$$

Furthermore, define the following operations on $\wp\left(A_{T}\right)$, for $i<3$ :

$$
C_{i} Q=\left\{p \in A_{T} \mid p \sim_{i} q \text { for some } q \in Q\right\} .
$$

Finally, we define the structure $\mathcal{D}_{\mathcal{G}, T}$ as:

$$
\mathcal{D}_{\mathcal{G}, T}=\left(\wp\left(A_{T}\right), \cup, \sim, \varnothing, C_{i}\right)_{i<3} .
$$

That is, $\mathcal{D}_{\mathcal{G}, T}$ is defined as the complex algebra of the structure $\left(A_{T}, \sim_{i}\right)_{i<3}$.

Since no confusion is likely to arise concerning the geometry involved, we usually omit the first subscript, writing $\mathcal{D}_{T}$ instead of $\mathcal{D}_{\mathcal{G}, T}$.

The name tripod stems from the observation that within the lattice of subspaces of $\mathcal{G}$, the subspace $H$ 'stands' on the three legs $S_{0}, S_{1}, S_{2}$. Observe that if $T=\left(H, S_{0}, S_{1}, S_{2}\right)$ is a tripod, then the dimension of $H$ cannot be smaller than two; hence, only geometries of dimension at least three can contain tripods. 
As we mentioned already informally, the algebras $\mathcal{D}_{\mathcal{G}, T}$ in the definition above form the link between slender Lyndon algebras and representable diagonal-free cylindric algebras. To start with, observe that for any tripod $T$ in a geometry $\mathcal{G}$, the algebra $\mathcal{D}_{\mathcal{G}, T}$ is of the similarity type of diagonal-free algebras. In fact, we know much more about $\mathcal{D}_{\mathcal{G}, T}$, as the next lemma will show.

Lemma 3.3 Suppose $T$ is a tripod in the projective geometry $\mathcal{G}$. Then $\mathcal{D}_{T}$ is isomorphic to a representable diagonal-free cylindric algebra of dimension three.

Proof. Let $\mathcal{G}=(P, L, I)$ be a geometry and suppose that $T$ is a tripod of $\mathcal{G}$. We will prove that $\mathcal{D}_{T}$ is a complete and atomic $\mathrm{Df}_{3}$ with rectangular atoms. This suffices to prove the Lemma by Fact 2.3(3).

It is easy to see that $\mathcal{D}_{T}$ is complete and atomic, since its boolean reduct is the power set algebra of $A_{T}$. The other properties of $\mathcal{D}_{T}$ will be proved in the two Claims below. As indicated above, in the proofs of these Claims the atom structure $\left(A_{T}, \sim_{i}\right)_{i<3}$ of $\mathcal{D}_{T}$ plays a crucial role.

Claim 1. $\mathcal{D}_{T}$ belongs to $\mathrm{Df}_{3}$.

Proof of Claim. It is well-known (cf. [Henkin, Monk \& Tarski 1971], Theorem 2.7.40 and its proof) and in fact, easy to see, that the complex algebra of any structure $\left(W, R_{1}, R_{2}, R_{3}\right)$ belongs to $\mathrm{Df}_{3}$ if and only if the relations $R_{i}$ are mutually commuting equivalence relations. (We say that two relations $R_{1}$ and $R_{2}$ commute if $R_{1}\left|R_{2}=R_{2}\right| R_{1}$, where | denotes relational composition). Since $\mathcal{D}_{T}$ is the complex algebra of the structure $\left(A_{T}, \sim_{i}\right)_{i<3}$, it suffices for us to prove that each $\sim_{i}$ is an equivalence relation and that for each pair of distinct $i$ and $j$, the relations $\sim_{i}$ and $\sim_{j}$ commute.

Let us first establish that $\sim_{i}$ is an equivalence relation for each $i<3$. Reflexivity and symmetry are easily proved, so we concentrate on transitivity. Suppose that $p \sim_{i} q \sim_{i} r$; that is, there are points $s$ and $s^{\prime}$ in $S_{i}$ such that $p, q$ and $s$ and $q, r$ and $s^{\prime}$ are collinear, respectively. If $p, q$ and $r$ are collinear then by Fact 2.1(6) the points $s$ and $s^{\prime}$ must be identical; it is then immediate that $p \sim_{i} r$. Now assume that $p, q$ and $r$ are not collinear and consider the projective space $\langle p, q, r\rangle$ which then must be a plane, cf. the picture below.

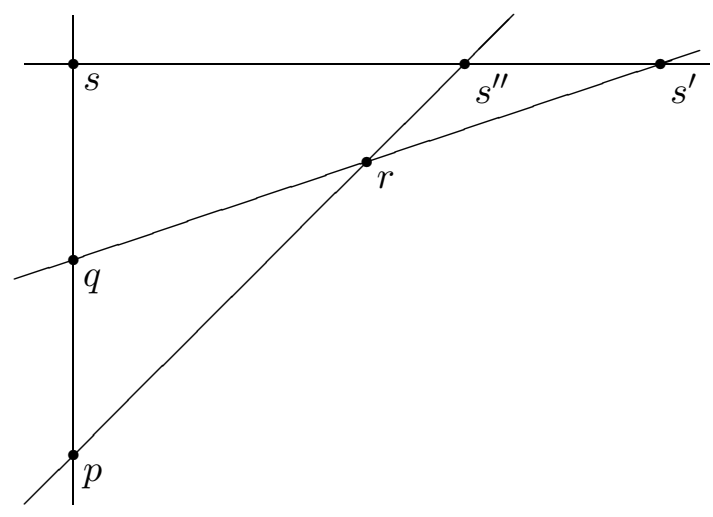


Since both $s$ and $s^{\prime}$ are elements of this plane, the line $\overline{s s^{\prime}}$ is a subset of $\langle p, q, r\rangle$ by the definition of a subspace. In a similar way, the line $\overline{p r}$ forms part of $\langle p, q, r\rangle$. By Fact 2.1(1), the lines $\overline{s s^{\prime}}$ and $\overline{p r}$ meet at some point $s^{\prime \prime}$ which is an element of the subspace $S_{i}$ since $\overline{s s^{\prime}} \subseteq S_{i}$. Since $p, r$ and $s^{\prime \prime}$ are collinear, this gives $p \sim_{i} r$.

Let us now check the commutativity condition. Obviously, it suffices to prove that for any $p, q$ and distinct $i$ and $j$ we have

$$
p \sim_{i} \mid \sim_{j} q \text { iff } p \text { and } q \text { are collinear with some } s \in S_{i} \bullet S_{j} .
$$

For, it readily follows from its definition (2) that $\bullet$ is a commutative operation; but from $S_{i} \bullet S_{j}=S_{j} \bullet S_{i}$ and (9) it is immediate that $\sim_{i}$ and $\sim_{j}$ commute.

In order to prove (9), suppose that $p \sim_{i} r \sim_{j} q$ for some $r \in A_{T}$. We first take care of the cases that $p, q$ and $r$ are not all distinct. If $p=q$ or $q=r$ then $p, q$ and $s_{i}$ are collinear and hence (9) follows from $s_{i} \in S_{i} \subseteq S_{i} \bullet S_{j}$. If $p=r$ then $p, q$ and $s_{j}$ are collinear; now we prove (9) by $s_{j} \in S_{j} \subseteq S_{i} \bullet S_{j}$.

Now assume that $p, q$ and $r$ are distinct. We claim that $p, q$ and $r$ cannot be collinear; for, if they were collinear, by Fact 2.1(6) the intersection of this line with $H$ would be a single point. Since both $s_{i}$ and $s_{j}$ belong to this intersection, we would obtain that $s_{i}=s_{j}$, which gives the desired contradiction. But if $p, q$ and $r$ are not collinear, then the projective space $\langle p, q, r\rangle$ is a plane. The lines $\overline{s_{i} s_{j}}$ and $\overline{p q}$ are part of $\langle p, q, r\rangle$, by the definition of a subspace. Hence, by Fact 2.1(1) these lines must meet, say, in $s$ (cf. the picture below).

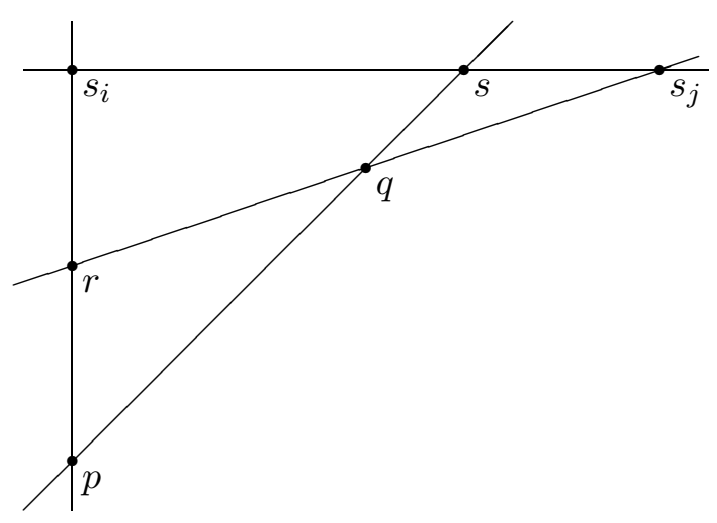

Clearly, $s$ is the point that we are looking for, since it is collinear with $p$ and $q$ and belongs to the subspace $S_{i} \bullet S_{j}$.

For the reverse direction of (9), assume that $p$ and $q$ are collinear with some point $s \in$ $S_{i} \bullet S_{j}$. Assume that $p$ and $q$ are distinct (if they are not distinct then we have $p \sim_{i} \mid \sim_{j} q$ by $p \sim_{i} p \sim_{j} q$ ). Now since $s \in S_{i} \bullet S_{j}$, there are three possibilities: either $s \in S_{i}$ or $s \in S_{j}$ or $s$ lies on some line $\overline{s_{i} s_{j}}$ with $s_{i} \in S_{i}, s_{j} \in S_{j}$.

In the first case we immediately see that $p \sim_{i} q$, whence $p \sim_{i} \mid \sim_{j} q$ by $q \sim_{j} q$; the second case goes by a symmetric argument. In the third case, consider the plane through the points $p, q, s, s_{i}$ and $s_{j}$ (cf. the same picture). By Fact 2.1(1), the lines $\overline{p s_{i}}$ and $\overline{q s_{j}}$ meet in some 
point, say $r$. We claim that $r$ does not lie in $H$, for suppose otherwise. By Fact 2.1(6), $s_{i}$ is the only point on $\overline{p s_{i}}$ that belongs to $H$, so we would find that $r=s_{i}$. Analogous reasoning concerning $\overline{q s_{j}}$ would yield that $r=s_{j}$. But this would imply that $s_{i}=s_{j}$ which cannot be the case by our assumption on the disjointness of $S_{i}$ and $S_{j}$. Hence, we may assume that $r$ belongs to $A_{T}$. By definition of $\sim_{i}$, the collinearity of $q, r$ and $s_{i}$ implies that $p \sim_{i} r$; and likewise, the collinearity of $q, r$ and $s_{j}$ implies that $r \sim_{j} q$. This shows that indeed $p \sim_{i} \mid \sim_{j} q$ and hence proves (9).

Claim 2. $\mathcal{D}_{T}$ is rectangularly atomic.

Proof of Claim. Finally, we turn to the proof that all atoms of $\mathcal{D}_{T}$ are rectangular. As with the other properties, rectangularity of the atoms of a $\mathrm{Df}_{3}$ corresponds to a property of the atom structure. We claim that it suffices to prove the following, for all points $p, q$ and $r$;

$$
\text { if } p \sim_{i} q \sim_{j} r \sim_{k} p \text { for distinct } i, j, k<3 \text {, then } p=q=r .
$$

For, using (10) we can reason as follows (without any further reference to the geometric origin of the algebra $\mathcal{D}_{T}$ or its atom structure). Consider an arbitrary atom of $\mathcal{D}_{T}$. By definition of $\mathcal{D}_{T}$, this atom must be a singleton, say $\{p\}$, for some point $p$ in $A_{T}=P \backslash H$. First we show that

$$
C_{0}\{p\}=C_{0} C_{1}\{p\} \cap C_{0} C_{2}\{p\} .
$$

For, suppose that $q \in C_{0} C_{1}\{p\} \cap C_{0} C_{2}\{p\}$. Then by the definition of $C_{0}$ and $C_{1}$, there exist $s$ and $t$ in $A_{T}$ such that $p \sim_{0} s \sim_{1} q$ and $p \sim_{0} t \sim_{2} q$, respectively; it follows that $q \sim_{1} s \sim_{0} t \sim_{2} q$. Therefore, $q=s$ by (10). Hence, $q \in C_{0}\{p\}$, since $s \in C_{0}\{p\}$. The opposite inclusion can be easily derived using the fact that the axioms $\mathrm{C} 2$ and $\mathrm{C} 4$ hold in $\mathcal{D}_{T}$. This proves (11), which places us in the right position to prove that $\{p\}$ is a rectangular atom:

$$
\{p\}=C_{0} C_{1}\{p\} \cap C_{0} C_{2}\{p\} \cap C_{1} C_{2}\{p\} .
$$

First, take an arbitrary point $q$ in $C_{0} C_{1}\{p\} \cap C_{0} C_{2}\{p\} \cap C_{1} C_{2}\{p\}$. Applying (11), we find that $q \in C_{0}\{p\} \cap C_{1} C_{2}\{p\}$. Then by the definition of the $C_{i}$ operations, there exists $r$ such that $p \sim_{0} q \sim_{2} r \sim_{1} p$; hence, by (10) $p$ and $q$ must be identical, showing that $q$ belongs to $\{p\}$. The opposite inclusion is easily proved using axiom $\mathrm{C} 2$.

The only thing left to do is to check condition (10); here we will use the geometric property of tripods that there are no collinear points belonging to the three spanning subspaces. Suppose that for some distinct $i, j, k<3$, we have that $p \sim_{i} q \sim_{j} r \sim_{k} p$ while $p$ and $q$ are distinct. Note that in this case, $r$ cannot be identical to both $p$ and $q$; without loss of generality, assume that $r \neq q$. We may thus freely speak of the lines $\overline{p q}$ and $\overline{q r}$.

By Fact 2.1(6), it follows from $p \sim_{i} q$ that the intersection of the line $\overline{p q}$ with $H$ is some point $s_{i} \in S_{i}$, and from $q \sim_{j} r$ that the intersection of the line $\overline{q r}$ with $H$ is some point $s_{j} \in S_{j}$. But since $S_{i} \cap S_{j}=\varnothing$, the points $s_{i}$ and $s_{j}$ are distinct. From this it follows that the lines $\overline{p q}$ and $\overline{q r}$ must be distinct; in particular, we have that $p$ and $r$ must be distinct, and that $p, q$ and $r$ can not be collinear. But then the subspace $\langle p, q, r\rangle$ is a plane. 
Now the intersection $S$ of this plane with $H$ must be a subspace. Since $S$ contains the points $s_{i}=\overline{p q} \cap H$ and $s_{j}=\overline{q r} \cap H$, it contains the line $\overline{s_{i} s_{j}}$. However, the plane $\langle p, q, r\rangle$ is not contained in $H$, for instance, $p \notin H$; therefore, $S$ cannot be bigger than a line. We conclude that $S$ must be identical to the line $\overline{s_{i} s_{j}}$. Now consider the line $\overline{r p}$; this line must intersect $H$ in a point $s_{k} \in S_{k}$, since $r \sim_{k} p$. But since $s_{k}$ also belongs to the plane $\langle p, q, r\rangle$ we find $s_{k} \in S$. By our previous observation then, $s_{k}$ must be incident with the line $\overline{s_{i} s_{j}}$, contradicting the tripod condition that there are no collinear points $s_{0} \in S_{0}, s_{1} \in S_{1}$ and $s_{2} \in S_{2}$.

Having proved these two claims, we may conclude by Fact 2.3(3) that indeed, $\mathcal{D}_{T}$ is a representable $\mathrm{Df}_{3}$.

QED

The next Lemma works the other way around. It states that for a 'small' set $\operatorname{Df}_{3} \mathcal{A}$ and an infinite geometry $\mathcal{G}$ of dimension at least three, we can find a tripod $T$ in $\mathcal{G}$ such that $\mathcal{A}$ can be embedded in $\mathcal{D}_{T}$.

Lemma 3.4 Let $\mathcal{A}$ be a countably based set $\mathrm{Df}_{3}$, and let $\mathcal{G}$ be an infinite projective geometry of dimension at least three. Then there is a tripod $T$ in $\mathcal{G}$ such that $\mathcal{A}$ can be embedded in $\mathcal{D}_{T}$.

Proof. Our aim is to find a tripod $T$ in $\mathcal{G}$ such that $\mathcal{D}_{T}$ is simple (that is, it is isomorphic to a set $\mathrm{Df}_{3}$ ) and each equivalence class of each relation $\sim_{i}$ is infinite.

Let $M$ be a basis of $\mathcal{G}$. Since $\mathcal{G}$ is an infinite geometry, there are two (not necessarily exclusive) possibilities: $\mathcal{G}$ may be of infinite order, or of infinite dimension. Observe that in the second case $M$ will be infinite as well. Fix some $t \in M$ and define $H$ as the subspace generated by $M \backslash\{t\}$; it is easy to see that $H$ is a hyperplane. By our assumption concerning the dimension of $\mathcal{G}$, we have that $|M \backslash\{t\}| \geq 3$. Partition $M \backslash\{t\}$ into three non-empty sets $M_{0}, M_{1}, M_{2}$, with the proviso that if $M$ is infinite, then each $M_{i}$ is infinite as well. We define $S_{i}$ to be the span of $M_{i}: S_{i}=\left\langle M_{i}\right\rangle$. Clearly, $H=\left\langle S_{0}, S_{1}, S_{2}\right\rangle$. Furthermore, it follows from Fact 2.1(7) that there is no triple of collinear points $s_{0} \in S_{0}, s_{1} \in S_{1}$ and $s_{2} \in S_{2}$. Hence, $T=\left(H, S_{0}, S_{1}, S_{2}\right)$ is a tripod in $\mathcal{G}$; let $A_{T}, C_{i}$ and $\mathcal{D}_{T}$ be as in Definition 3.2. Our main aim is now to prove the following Claims.

Claim 1. $\mathcal{D}_{T}$ is simple.

Proof OF Claim. It suffices to show that

for any $p, q \in A_{T}$, there are $v, u \in A_{T}$ such that $p \sim_{0} v \sim_{1} u \sim_{2} q$.

For, assume that (13) holds and take an arbitrary non-empty subset $Q$ of $A_{T}=P \backslash H$, and an arbitrary point $p \in A_{T}$. Since $Q$ is non-empty it contains some point $q$. Then (13) yields points $v$ and $u$ in $A_{T}$ such that $p \sim_{0} v \sim_{1} u \sim_{2} q$; from this and the definitions we may easily infer that $p \in C_{0} C_{1} C_{2} Q$. But since $p$ was arbitrary, this shows that $C_{0} C_{1} C_{2} Q=A_{T}$ whence $\mathcal{D}_{T}$ is simple indeed, by Fact 2.3(1).

In order to prove (13), let $p$ and $q$ be arbitrary points in $A_{T}$. If $p=q$ then (13) trivially follows by reflexivity of the relations $\sim_{i}$. Now assume that $p \neq q$; hence, we may speak of the line $\overline{p q}$. 
One of the key observations is that since $H$ is a hyperplane, it must intersect the line $\overline{p q}$ by Fact 2.1(2). By Fact 2.1(6) there must be a unique intersection point, say $h$. Note that $H=\left\langle S_{0}, S_{1}, S_{2}\right\rangle=\left\langle S_{0},\left\langle S_{1}, S_{2}\right\rangle\right\rangle=S_{0} \bullet\left(S_{1} \bullet S_{2}\right)$ (cf. Fact 2.1, parts (5) and (4), respectively). Now define $S_{12}$ to be the subspace $S_{1} \bullet S_{2}$, and let $\sim_{12}$ be the corresponding relation; that is, $p \sim_{12} q$ iff $p$ and $q$ are collinear with some $s \in S_{12}$. Observe that it follows from (9) in the proof of the previous Lemma that $\sim_{12}$ is the relational composition of $\sim_{1}$ and $\sim_{2}$.

Since $h \in H=S_{0} \bullet\left(S_{1} \bullet S_{2}\right)$ it follows, again from (9), that $p \sim_{0} \mid \sim_{12} q$. Together with the observation that $\sim_{12}=\sim_{1} \mid \sim_{2}$ this proves (13).

Claim 2. For each $i$, the equivalence class of $\sim_{i}$ is infinite.

Proof of Claim. Let $E$ be an $\sim_{i}$-equivalence class. We now distinguish cases as to the reason why $\mathcal{G}$ is infinite. If $\mathcal{G}$ is of infinite order, consider a point $p$ from $E$ and the line $\overline{p s_{i}}$ for some point $s_{i} \in S_{i}$. Note that $s_{i}$ is the only point on this line which belongs to $H$, by Fact 2.1(6). All other points of the line $\overline{p s_{i}}$ belong to the $\sim_{i}$-equivalence class $E$, and as there are infinitely many of these points, $E$ is infinite.

If, on the other hand, the dimension of $\mathcal{G}$ is infinite, we reason as follows. Take an arbitrary point $p$ of $E$ and consider the set $B$ of all lines connecting $p$ to some point in $S_{i}$. Observe that two distinct lines in $B$ cannot intersect in any point other than $p$. It is thus obvious that the function mapping a line in $B$ to the (unique!) point where the line intersects $S_{i}$, is a bijection from $B$ onto $S_{i}$. In particular, this shows $B$ to be infinite, since $S_{i}$ is infinite by our proviso on $M_{i}$. But since any line must contain at least three points, we may choose on each line in $B$ a point which is distinct from $p$ and not in $S_{i}$. The collection of these points forms then an infinite subset of $E$. Hence, $E$ itself must be infinite.

How can we prove the Lemma from these Claims? To start with, the proof of the previous Lemma establishes that $\mathcal{D}_{T}$ is a complete and atomic representable $\mathrm{Df}_{3}$. And second, by Claim 1, $\mathcal{D}_{T}$ is simple. It thus follows from Fact 2.3(3) that $\mathcal{D}_{T}$ is isomorphic to some full set $\operatorname{Df}_{3}$, say, $\mathcal{B}$. Suppose that $\mathcal{B}$ is based on the sets $\left\{Y_{i}: i<3\right\}$. It is not difficult to see that each $Y_{i}$ must be infinite. For, if $\mathcal{D}_{T}$ and $\mathcal{B}$ are isomorphic, then so must be their underlying relational structures $\left(A_{T}, \sim_{i}\right)_{i<3}$ and $\left(\prod_{i<3} Y_{i}, \equiv_{i}\right)$. But then it follows from Claim 2 that each $\equiv_{i}$-equivalence class is infinite; from this it is immediate that each $Y_{i}$ is infinite as well.

Now recall that $\mathcal{A}$ is a countably based three-dimensional diagonal-free cylindric set algebra; suppose its base system is $\left\{U_{i}: i<3\right\}$, where each $U_{i}$ is countable. Hence, for each $i<3$, $\left|U_{i}\right| \leq\left|Y_{i}\right|$ since $Y_{i}$ is infinite. Then we may apply Fact 2.3(4) and conclude that $\mathcal{A}$ can be embedded in $\mathcal{B}$, and therefore, in $\mathcal{D}_{T}$, by isomorphism.

QED

In order to put the above two lemmas to work we now specify a translation mapping $D f_{3}$-equations to universal formulas in the language of slender Lyndon algebras.

Definition 3.5 Let $\mathrm{h}, \mathrm{s}_{i},(i<3)$, be distinguished variables in the language of slender Lyndon algebras. The translation ${ }^{-}$, mapping $D f_{3}$-terms to $S L$-terms, is defined by the following term induction:

$$
\bar{x}=x \cdot-\mathrm{h}
$$




$$
\begin{aligned}
\overline{-\sigma} & =-\bar{\sigma} \cdot-\mathrm{h} \\
\overline{\sigma \cdot \tau} & =\bar{\sigma} \cdot \bar{\tau} \\
\overline{\mathrm{c}_{i} \sigma} & =\left(\bar{\sigma} ; \mathrm{s}_{i}\right) \cdot-\mathrm{h}
\end{aligned}
$$

Furthermore, define the following 'subspace-equation'

$$
\operatorname{subspace}(\sigma)=\sigma ; \sigma \approx \sigma,
$$

and the 'tripod-formula'

$$
\begin{aligned}
\operatorname{tripod}\left(\mathrm{h}, \mathrm{s}_{0}, \mathrm{~s}_{1}, \mathrm{~s}_{2}\right)= & \mathrm{h} \not 1 \wedge \bigwedge_{0 \leq i<3}\left(\mathrm{~s}_{i} \not \approx 0\right) \wedge \bigwedge_{0 \leq i<3}\left(\mathrm{~s}_{i} \prec \mathrm{h}\right) \wedge \bigwedge_{0 \leq i, j<3, i \neq j}\left(\mathrm{~s}_{i} \cdot \mathrm{s}_{j} \approx 0\right) \wedge \\
& \wedge \text { subspace }(\mathrm{h}) \wedge \bigwedge_{0 \leq i<3} \operatorname{subspace}\left(\mathrm{s}_{i}\right) \wedge \\
& \wedge \mathrm{s}_{0} \cdot\left(\mathrm{s}_{1} ; \mathrm{s}_{2}\right) \approx 0 \wedge\left(\mathrm{s}_{0} ; \mathrm{s}_{1}\right) ; \mathrm{s}_{2} \approx h .
\end{aligned}
$$

Finally, we take the translation $\operatorname{tr}(\sigma \approx \tau)$ of a $D f_{3}$-equation $\sigma \approx \tau$ to be the formula

$$
\operatorname{tr}(\sigma \approx \tau)=\operatorname{tripod}\left(\mathrm{h}, \mathrm{s}_{0}, \mathrm{~s}_{1}, \mathrm{~s}_{2}\right) \rightarrow \bar{\sigma} \approx \bar{\tau}
$$

Observe that the translation of a $D f_{3}$-equation is a boolean combination of $S L$-equations and thus a universal formula. The precise meaning of the translation will be discussed in the proof of Lemma 3.6 below.

Recall that $\mathrm{RDf}_{3}$ denotes the class of representable diagonal-free cylindric algebras, and $\mathrm{S}(\mathrm{G})$ denote the class of slender Lyndon algebras associated with a class $\mathrm{G}$ of geometries.

Lemma 3.6 Let $\mathrm{G}$ be a class of projective geometries which contains an infinite projective geometry of dimension at least three. Then for any pair of $D f_{3}$-terms $\sigma$ and $\tau$,

$$
\mathrm{RDf}_{3} \models \sigma \approx \tau \text { iff } \mathrm{S}(\mathrm{G}) \models \operatorname{tr}(\sigma \approx \tau) .
$$

Proof. Two main observations underpin our proof of this Lemma. Let $\mathcal{G}=(P, L, I)$ be a geometry and consider subsets $H, S_{0}, S_{1}$ and $S_{2}$ of $P$. Assume that $H, S_{0}, S_{1}$ and $S_{2}$ interpret the variables $h, s_{0}, s_{1}$ and $s_{2}$, respectively. Our first observation is that

$$
\operatorname{tripod}\left(H, S_{0}, S_{1}, S_{2}\right) \text { holds in } \mathcal{S}(\mathcal{G}) \text { iff }\left(H, S_{0}, S_{1}, S_{2}\right) \text { is a tripod of } \mathcal{G} \text {. }
$$

And second, if we assume that $T=\left(H, S_{0}, S_{1}, S_{2}\right)$ is a tripod in $\mathcal{G}$, we have for any term $\sigma$ :

$$
\sigma^{\mathcal{D}_{T}}\left(Q_{1} \cap A_{T}, \ldots, Q_{n} \cap A_{T}\right)=\bar{\sigma}^{\mathcal{S}(\mathcal{G})}\left(Q_{1}, \ldots, Q_{n}, H, S_{0}, S_{1}, S_{2}\right),
$$

for all $Q_{1}, \ldots, Q_{n} \in \wp(P)$. The details of this proof are immediate by the definitions and therefore left to the reader - the crucial case of the inductive step follows from the observation that

$$
C_{i} Q=\left(S_{i} \bullet Q\right) \cap A_{T},
$$

as a direct proof reveals. 
The proof of (14) is straightforward as well, but let us briefly discuss the contributions of the respective conjuncts. The important thing to remember is that the operation symbol; is interpreted as the operation $\bullet$, and that for subspaces $X$ and $Y, X \bullet Y$ denotes the subspace spanned by $X$ and $Y$, cf. Fact 2.1(4).

Now the first conjuncts of the tripod-formula obviously have the following meaning:

$$
\begin{array}{ll}
\mathrm{h} \not \approx 1 & H \text { is a proper subset of } P, \\
\bigwedge_{0 \leq i<3}\left(\mathrm{~s}_{i} \not \approx 0\right) & \text { each } S_{i} \text { is non-empty, } \\
\bigwedge_{0 \leq i<3}\left(\mathrm{~s}_{i} \prec \mathrm{h}\right) & \text { each } S_{i} \text { is a proper subset of } H, \\
\bigwedge_{0 \leq i, j<3, i \neq j}\left(\mathrm{~s}_{i} \cdot \mathrm{s}_{j} \approx 0\right) & S_{0}, S_{1} \text { and } S_{2} \text { are mutually disjoint, }
\end{array}
$$

The conjuncts subspace(h) and $\bigwedge_{0 \leq i<3}$ subspace $\left(\mathrm{s}_{i}\right)$ state that $H, S_{0}, S_{1}$ and $S_{2}$ are subspaces of the geometry; this follows from the observation that for any subset $X$ of $P$

$$
X \text { is a subspace of } \mathcal{G} \text { iff } X=X \bullet X \text {. }
$$

Then, the formula $\left(\mathrm{s}_{0} ; \mathrm{s}_{1}\right) ; \mathrm{s}_{2} \approx h$ denotes that $H$ is spanned by $S_{0}, S_{1}$ and $S_{2}$. Finally, given the disjointness of $S_{0}, S_{1}$ and $S_{2}$, the conjunct $\mathrm{s}_{0} \cdot\left(\mathrm{s}_{1} ; \mathrm{s}_{2}\right) \approx 0$ holds in $\mathcal{S}(\mathcal{G})$ if and only if there are no three collinear points from $S_{0}, S_{1}$ and $S_{2}$, respectively. This proves (14).

In order to prove the Lemma, first suppose that the formula $\operatorname{tr}(\sigma \approx \tau)$ is not valid in the class $\mathrm{S}(\mathrm{G})$. Then there is some geometry $\mathcal{G}$ in $\mathrm{G}$ such that $\operatorname{tr}(\sigma \approx \tau)$ does not hold in $\mathcal{S}(\mathcal{G})$; that is, there are $H, S_{0}, S_{1}, S_{2}, \vec{Q}$ such that $\operatorname{tripod}\left(H, S_{0}, S_{1}, S_{2}\right)$ holds in $\mathcal{S}(\mathcal{G})$ while $\bar{\sigma} \mathcal{S}(\mathcal{G})\left(\vec{Q}, H, S_{0}, S_{1}, S_{2}\right) \neq \bar{\tau} \mathcal{S}(\mathcal{G})\left(\vec{Q}, H, S_{0}, S_{1}, S_{2}\right)$. It follows from $(14)$ that $T=\left(H, S_{0}, S_{1}, S_{2}\right)$ is a tripod in $\mathcal{G}$; thus, by Lemma $3.3 \mathcal{D}_{T}$ is a representable diagonal-free cylindric algebra of dimension 3. With the help of (15) we may also infer that $\sigma^{\mathcal{D}_{T}}\left(Q_{1} \cap A_{T}, \ldots, Q_{n} \cap A_{T}\right) \neq$ $\tau^{\mathcal{D}_{T}}\left(Q_{1} \cap A_{T}, \ldots, Q_{n} \cap A_{T}\right)$. But then we have shown that the equation $\sigma \approx \tau$ is not valid in the class of representable $\mathrm{Df}_{3}$ 's.

For the opposite direction, assume that the equation $\sigma \approx \tau$ is not valid in the class of representable $\mathrm{Df}_{3}$ 's. By Fact 2.3(5) we may assume that the equation can be refuted in some diagonal-free cylindric set algebra $\mathcal{A}$ with unit $V=Y_{0} \times Y_{1} \times Y_{2}$ for some non-empty, countable sets $Y_{i}, i<3$. By Lemma 3.4, this algebra $\mathcal{A}$ can be embedded in the structure $\mathcal{D}_{\mathcal{G}, T}$, where $\mathcal{G}$ is any infinite geometry in $\mathrm{S}(\mathrm{G})$ of dimension at least three, and $T=\left(H, S_{0}, S_{1}, S_{2}\right)$ is an appropriate tripod in $\mathcal{G}$. It follows that we can also refute $\sigma \approx \tau$ in $\mathcal{D}_{T}$, so $\sigma^{\mathcal{D}_{T}}(\vec{Q}) \neq \tau^{\mathcal{D}_{T}}(\vec{Q})$ for some $\vec{Q} \subseteq A_{T}$. By $(14), \operatorname{tripod}\left(H, S_{0}, S_{1}, S_{2}\right)$ holds in $\mathcal{S}(\mathcal{G})$ since $\left(H, S_{0}, S_{1}, S_{2}\right)$ is a tripod in $\mathcal{G}$. Applying (15), we get $\bar{\sigma} \mathcal{S}(\mathcal{G})\left(\vec{Q}, H, S_{0}, S_{1}, S_{2}\right) \neq \bar{\tau} \mathcal{S}(\mathcal{G})\left(\vec{Q}, H, S_{0}, S_{1}, S_{2}\right)$. So $\operatorname{tr}(\sigma \approx \tau)$ does not hold in $\mathcal{S}(\mathcal{G})$. $\quad$ QED

We are now in a position to bring these Lemmas together and prove our main technical result.

Proof of Theorem 3.1. Suppose $G$ is a class of geometries which contains an infinite geometry of dimension $d \geq 3$. Let $\sigma \approx \tau$ be a $D f_{3}$-equation. By the previous Lemma, $\sigma \approx \tau$ is valid in the class of representable $\mathrm{Df}_{3}$ 's iff $\operatorname{tr}(\sigma \approx \tau)$ is valid in the class of Lyndon algebras over $\mathrm{G}$. We already say that the translation $\operatorname{tr}$ turns $D f_{3}$-equations into universal formulas in the language of slender Lyndon algebras. Now it is relatively simple to show that $S(G)$ is 
a discriminator class: the unary discriminator term is $x ; 1$. (All that is required is to show that for every non-empty set $Q$ of points in a geometry $\mathcal{G}=(P, L, I)$ we have $Q \bullet P=P$, and this is straightforward.) Hence, by Fact 2.2 this means that there is a recursive procedure of rewriting universal formulas into equivalent equations of slender Lyndon algebras. So the equational theory of representable $\mathrm{Df}_{3}$ 's reduces to the equational theory of $\mathrm{S}(\mathrm{G})$. By a result of R. Maddux, the first-mentioned theory is undecidable, cf. [Maddux 1980]; hence, the equational theory of $\mathrm{S}(\mathrm{G})$ is undecidable as well.

QED

Finally, as we mentioned in the introduction to this paper, S. Givant pointed out to us that he could obtain the following strengthenings of our Theorem 1:

1. If $\mathrm{G}$ is a class of projective geometries such that for each natural number $n$ there is a geometry in $\mathrm{G}$ of size at least $n$ and dimension at least three, then the equational theory of LG is undecidable.

2. If an arbitrary class of relation algebraas contains, for each positive integer $n$, a Lyndon algebra of dimension at least $n$, then the equational theory of the class is undecidable.

The proof of the first statement is analogous to the proof of our Theorem 1; Givant proves and uses an undecidability result for classes of $\mathrm{Df}_{3}$ 's such that for each natural number $n$, the class contains a set algebra with base sets each of size at least $n$. The proof of the second statement uses ideas developed in [Andréka, Givant \& Németi 1997].

\section{References}

[Andréka, Givant \& Németi 1997] H. Andréka, S. Givant and I. Németi, Decision Problems for Equational Theories of Relation Algebras, Memoirs of the American Mathematical Society, 1997.

[Garner 1981] L. Garner, An Outline of Projective Geometry, Elsevier North Holland, Amsterdam, 1981.

[Givant 199?] S. Givant, "Universal classes of simple relation algebras", Journal of Symbolic Logic, to appear.

[Henkin, Monk \& Tarski 1971] L. Henkin, J.D. Monk and A. Tarski, Cylindric Algebras Part 1 North-Holland, Amsterdam, 1971.

[Henkin, Monk \& Tarski 1985] L. Henkin, J.D. Monk and A. Tarski, Cylindric Algebras Part 2, North-Holland, Amsterdam, 1985.

[Jipsen 1993] P. Jipsen, "Discriminator varieties of Boolean algebras with residuated operators", in: [Rauszer 1993], pp. 239-252.

[Jónsson 1959] B. Jónsson, "Representation of modular lattices and of relation algebras", Transactions of the American Mathematical Society, 92 (1959), pp. 449-464. 
[Jónsson 1982] B. Jónsson, "Varieties of relation algebras", Algebra Universalis, 15 (1982), pp. 273-298.

[Jónsson \& Tarski 1952] B. Jónsson and A. Tarski, "Boolean algebras with operators, Part 2", American Journal of Mathematics, 74 (1952), pp. 127-162.

[Lyndon 1961] R. Lyndon, "Relation Algebras and projective geometries", Michigan Mathematics Journal, 8 (1961), pp. 21-28.

[Maddux 1980] R. Maddux, "The equational theory of $C A_{3}$ is undecidable", J. Symbolic Logic, 45 (1980), pp. 311-316.

[Marx \& Venema 1997] M. Marx and Y. Venema, Multi-Dimensional Modal Logic, Kluwer Academic Press, Dordrecht, 1997.

[Monk 1964] J.D. Monk, "On representable relation algebras", Michigan Mathematical Journal, 11 (1964), pp. 207-210.

[Németi 1991] I. Németi, "Algebraization of quantifier logics, an introductory overview", Studia Logica, 50, (1991), pp.485-569.

[Rauszer 1993] C. Rauszer (ed.), Algebraic methods in logic and computer science, Banach Center Publications, Volume 28, Polish Academy of Sciences, Warsaw, 1993.

[Stebletsova 1998] V. Stebletsova, Modal logic of projective geometries of finite dimension, Technical Report 184, Department of Philosophy, Utrecht University, 1998.

[Tarski \& Givant 1988] A. Tarski and S. Givant, A formalization of set theory without variables, AMS Colloquium Publications, 41 (1988).

[Venema 1998] Y. Venema, Points, Lines and Diamonds: a Two-Sorted Modal Logic for Projective Planes, Technical report ML-1998-04, Institute for Logic, Language and Information, University of Amsterdam, 1998. (To appear in the Journal of Logic and Computation). 